Research Paper

\title{
Combination of plasma HA and circulating M2-like monocytes may serve as a diagnostic marker for breast
}

\section{cancer}

\author{
Boke Zhang ${ }^{1 *}$, Manlin $\mathrm{Cao}^{2 *}$, Yiqing He${ }^{1}$, Yiwen Liu ${ }^{1}$, Guoliang Zhang ${ }^{1}$, Cuixia Yang ${ }^{1}$, Yan Du ${ }^{1}$, Jing $\mathrm{Xu}^{3}$, \\ Jiajie $\mathrm{Hu}^{1}$, Feng $\mathrm{GaO}^{4 \bowtie}$ \\ 1. Department of Molecular Biology, Shanghai Jiao Tong University Affiliated Sixth People's Hospital, 600 Yishan Road, Shanghai 200233, PR China; \\ 2. Department of Rehabilitation Medicine, Shanghai Jiao Tong University Affiliated Sixth People's Hospital, 600 Yishan Road, Shanghai 200233, PR China; \\ 3. Department of Clinical Laboratory, Shanghai Jiao Tong University Affiliated Sixth People's Hospital, 600 Yishan Road, Shanghai 200233, PR China; \\ 4. Department of Molecular Biology and Clinical Laboratory, Shanghai Jiao Tong University Affiliated Sixth People's Hospital, 600 Yishan Road, Shanghai \\ 200233, PR China. \\ * Both are co-first authors
}

$\square$ Corresponding author: Feng Gao, Department of Molecular Biology and Clinical Laboratory, Shanghai Jiao Tong University Affiliated Sixth People's Hospital, Shanghai 200233, PR China. Tel: +86-21-64369181; Fax: +86-21-63701361; E-mail: gao3507@126.com

(1) Ivyspring International Publisher. This is an open access article distributed under the terms of the Creative Commons Attribution (CC BY-NC) license (https:// creativecommons.org/licenses/by-nc/4.0/). See http://ivyspring.com/terms for full terms and conditions.

Received: 2017.03.23; Accepted: 2017.08.28; Published: 2017.09.29

\begin{abstract}
Background: Breast cancer (BC)-derived hyaluronan (HA) can induce the formation of M2-like tumor-associated macrophages (TAMs) in tumor context. However, little is known about the correlation between circulating M2-like monocytes and plasma HA in BC patients. This study focused on evaluating the relationship between circulating M2-like monocytes and plasma $\mathrm{HA}$, and further appraised the diagnostic value of them in BC.

Methods: The expression of M2-like TAMs and HA was determined in pathological tissues by immunohistochemistry. Flow cytometry was used to detect the levels of circulating $\mathrm{CD} 14^{+} \mathrm{CD} 204^{+}$M2-like monocytes in $81 \mathrm{BC}$ patients, 45 patients with breast benign diseases, and 46 healthy subjects. The levels of HA, CEA, and CA15-3 were measured in plasma samples using chemiluminescence method.

Results: M2-like TAMs and HA expressions were elevated in BC tissues compared with benign tissues. In correspondence, the frequency of circulating $\mathrm{CD} 14^{+} \mathrm{CD} 204^{+} \mathrm{M} 2$-like monocytes and the plasma HA levels were significantly higher in patients with BC than those in control groups. Importantly, there was a positive correlation between circulating M2-like monocytes and the plasma HA (Spearman $r=0.404, p<0.001$ ). Area under receiver operating characteristic curve (ROC) for the combination of circulating M2-like monocytes and $\mathrm{HA}$ was 0.899 (95\% Cl: 0.853-0.946), which was higher than the panel of CEA and CA15-3.

Conclusions: The frequency of circulating $\mathrm{CD} 14^{+} \mathrm{CD} 204^{+} \mathrm{M} 2$-like monocytes was positively correlated to plasma HA levels. The combination of circulating $\mathrm{CD} 14^{+} \mathrm{CD} 204^{+} \mathrm{M} 2$-like monocytes and plasma HA could provide considerable diagnostic value in BC.
\end{abstract}

Key words: Breast cancer, CD163, CD204, biomarker, diagnosis

\section{Introduction}

Breast cancer $(\mathrm{BC})$ remains one of the most prevalent cancers worldwide and a leading cause of cancer-related mortality in China [1, 2]. Early detection and diagnosis are important to reduce mortality and improve long-term survival. Unfortunately, the best available approach by mammographic screening has several limitations that include the use of ionizing radiation and poor 
diagnostic accuracy. Tumor biomarkers in peripheral blood are effective and non-invasive screening tools. However, the frequently used biomarkers of breast cancer, including carcinoembryonic antigen (CEA) and cancer antigen 15-3 (CA15-3), are elevated most of the time in advanced stages and lack sensitivity at early stages [3]. Therefore, it is urgent to search for other biomarkers with high sensitivity for screening and diagnosis of breast cancer patients.

Macrophages are often found in the tumor stroma, where macrophages are induced to tumor-associated macrophages (TAMs) [4, 5]. Macrophages are heterogeneous in population and can be differentiated into M1-like and M2-like macrophages [4, 6]. According to phenotype, function, and expression of cytokines, TAMs usually resemble the M2-like macrophages, which promote tumor growth and progression in BC patients [7, 8]. Some microenvironmental stimuli are shown to influence the phenotype of TAMs in breast tumors. For example, several anti-inflammatory mediators, including IL-4, IL-10, IL-13, TGF- $\beta$, and M-CSF, lead to the formation of M2-like macrophages [4, 7]. However, tumor microenvironment includes both cellular and noncellular (matrix) components. Hyaluronan (HA) is one of the main extracellular matrix constituent. In breast cancer, increased levels of HA promote tumor progression and are correlated with tumor cell proliferation and migration $[9,10]$. Stromal-derived $\mathrm{HA}$ can serve as a microenvironmental signal for TAMs recruitment and remodel the local microenvironment to promote tumor angiogenesis in a mouse mammary tumor model [11]. Moreover, accumulation of HA facilitates macrophages infiltration in breast cancer tissue and HA fragments are capable of enhancing macrophage differentiation into a phenotype of M2-like macrophages [12-14]. Therefore, there is a close relationship between TAMs and HA in breast tumor microenvironment, but little is known about the correlation between them in peripheral blood of $\mathrm{BC}$ patients.

Recently, several studies suggested that M2-like TAMs in breast cancer tissue could migrate into peripheral blood to become circulating M2-like monocytes and facilitate breast tumor metastasis [15-17]. Furthermore, circulating M2-like monocytes are elevated in some cancers, such as lung cancer [18], colorectal cancer [19], and classical Hodgkin Lymphoma [20]. Therefore, circulating M2-like monocytes may serve as a new tumor biomarker for breast cancer. In our previous study, we have demonstrated that serum HA concentrations were elevated in breast cancer patients [10]. These findings suggest that circulating M2-like monocytes and HA constitute a potential panel combining biomarkers for breast cancer screening. In this study, we investigated the relationship of the circulating M2-like monocytes with plasma HA in BC patients, with the purpose of identifying the combined biomarkers for the diagnosis of breast cancer.

\section{Materials and Methods}

\section{Study subjects}

The study was conducted according to the revised Declaration of Helsinki, 2013. Informed consent was obtained from all participants and the study protocol was approved by the Ethics Committee of Shanghai Jiao tong University. Blood samples were collected from 81 breast cancer patients and the control groups, which contained 45 patients with benign breast disease and 46 age-matched healthy subjects. All patients were diagnosed by histological evaluation for the first time at Shanghai Cancer Center before they received any treatment. All healthy volunteers were also free of any medications and had no cancer, infectious diseases, and others. The clinical characteristics of patients and healthy subjects were shown in Table 1.

\section{Sample preparation and storage}

Two ml peripheral blood samples were drawn into EDTA tubes and transported on ice to the laboratory. Firstly, $100 \mu \mathrm{l}$ blood samples were stained with the fluorescently-labeled anti-human monoclonal antibodies and analyzed by flow cytometry within 24 hours. After staining, the residual blood was centrifuged at $1000 \times \mathrm{g}$ at $4^{\circ} \mathrm{C}$ for $10 \mathrm{~min}$ to collect plasma, which was transferred into plain polypropylene tubes and stored at $-20^{\circ} \mathrm{C}$ for further assay.

\section{Flow cytometry analysis}

To determine the frequency of the circulating M2-like monocytes, peripheral blood samples were labeled with $7.5 \mu 1$ FITC-anti-CD14 (Beckman Coulter, France) and $6 \mu 1$ PE-anti-CD204 (R\&D Systems, USA), and incubated for $15 \mathrm{~min}$ at room temperature in the dark. Subsequently, the blood cells were lysed and fixed with $2 \mathrm{ml}$ of FACS lysing solution. The isotype-matched controls of FITC-IgG1 and PE-IgG2a were used as negative controls. The circulating CD14 ${ }^{+} \mathrm{CD} 204^{+}$monocytes were detected using a FACS Navios flow cytometer (Beckman Coulter, USA) and data were analyzed with FlowJo software (Tree Star, Inc. USA). 
Table 1. The clinical characteristics of all subjects

\begin{tabular}{|c|c|c|c|}
\hline Characteristics & Healthy control $(n=46)$ & Benign group $(n=45)$ & Malignant group $(n=81)$ \\
\hline Age(year) & $49.80 \pm 7.45$ & $49.84 \pm 7.95$ & $53.09 \pm 9.72$ \\
\hline \multirow[t]{5}{*}{ Histology type (NO. } & & Adenosis $(20,44.4 \%)$ & Invasive ductal carcinoma $(54,66.7 \%)$ \\
\hline & & Fibroadenoma $(10,22.2 \%)$ & Invasive lobular carcinoma $(13,16.0 \%)$ \\
\hline & & Intraduct papilloma $(8,17.8 \%)$ & Ductal carcinoma in situ $(7,8.6 \%)$ \\
\hline & & Fiber epithelioma $(4,8.9 \%)$ & Intraductal papillary carcinoma $(5,6.2 \%)$ \\
\hline & & Others $(3,6.7 \%)$ & Others $(2,2.5 \%)$ \\
\hline WBC $\left(\times 10^{9} / \mathrm{L}\right)$ & $5.66 \pm 0.98$ & $5.98 \pm 1.12$ & $6.01 \pm 1.45$ \\
\hline Monocytes $\left(\times 10^{9} / \mathrm{L}\right)$ & $0.31 \pm 0.10$ & $0.35 \pm 0.09$ & $0.35 \pm 0.11$ \\
\hline
\end{tabular}

\section{Plasma concentrations of CA15-3, CEA, and HA}

The concentrations of plasma hyaluronan (HA; Maglumi 2000, China), carcinoembryonic antigen (CEA; Architect i2000SR, Abbott, USA), and cancer antigen 15-3 (CA15-3; cobas e601, Roche, Switzerland), were measured by chemiluminescence method according to the manufacturer's instructions.

\section{Immunohistochemistry}

Immunohistochemical staining of $\mathrm{HA}$ and CD204 were performed using the formalin-fixed, paraffin-embedded biopsy specimens of breast cancer and breast benign disease. Briefly, $5-\mu \mathrm{m}$ sections were deparaffinized, rehydrated and microwaved in sodium citrate for antigen-retrieval. The sections were blocked at room temperature for $1 \mathrm{~h}$ with $5 \%$ BSA in PBS. For HA staining, the slides were incubated with biotinylated HABP (Merck, Germany, dilution: 1:50) at $4^{\circ} \mathrm{C}$ overnight. Slides were then washed three times with PBS and incubated with Streptavidin-ABC for 30 min at room temperature. To detect CD204 expression, the slides were incubated with anti-human CD204 antibodies (Molecular Probes, USA, dilution: 1:100) overnight at $4^{\circ} \mathrm{C}$. Next day, the slides were rinsed with PBS before incubation with biotinylated secondary antibody (Boster, China) for 30 $\mathrm{min}$ at room temperature. After removing non-reacted secondary antibodies, the sections were incubated with Streptavidin-ABC and then developed with DAB Substrate Kit to visualize the immunolabeling.

\section{Statistical analysis}

All statistical analyses and figures were performed with SPSS 20.0 (IBM, USA). Unpaired t test or one-way ANOVA turkey's multiple comparisons were used to analyze normally distributed data (presented as the mean $\pm \mathrm{SD}$ ). Non-normally distributed values were expressed as a median (P25-P75), which were evaluated by the Mann-Whitney $U$ test or Kruskal-Wallis nonparametric test. Spearman rank correlation test was performed to evaluate the correlations between plasma levels of HA and circulating M2-like monocytes. The diagnostic efficiency of the individual markers and their combination was assessed by receiver operating curve (ROC) analysis. Logistic regression analysis was performed to obtain sensitivity/specificity of the optimal combination of circulating M2-like monocytes, HA, CEA, and CA 15-3. 2-sided $p$ value $<0.05$ was considered statistical significance in our study.

\section{Results}

\section{The M2-like macrophages and HA were elevated in breast cancer tissues}

We first used the CD204 as a biomarker for detecting M2-like macrophages in tissues of breast cancer and benign breast disease. The expressions of HA were also determined in the matched tissues. As shown in Fig.1, our result showed that CD204+ M2-like macrophages were accumulated in the stroma of breast cancer, which were higher than that in benign tissue. Furthermore, HA content was also increased in breast cancer tissue compared with benign tissue.

\section{Circulating M2-like monocytes and plasma HA were increased in patients with breast cancer}

Table 1 summarized the clinical characteristics of BC patients, patients with benign breast disease, and healthy controls. In the $81 \mathrm{BC}$ patients investigated, invasive ductal carcinoma $(80.2 \%)$ was the most frequently occurring cancer, followed by invasive lobular carcinoma $(7.4 \%)$ and ductal carcinoma in situ (6.2\%). Moreover, $42.2 \%$ of the benign disease was adenosis, followed by fibroadenoma $(26.7 \%)$ and intraduct papilloma (17.8\%). Furthermore, the distribution of age, the counts of WBC and monocytes in peripheral blood were not significantly different between the breast cancer, benign, and healthy groups.

In our study, plasma HA levels were significantly increased in breast cancer group (61.63 (52.04-81.50) $\mathrm{ng} / \mathrm{ml}$ ) compared with control group $(49.13(45.08-55.91) \mathrm{ng} / \mathrm{ml}), p<0.001$, Figure 3A). $\mathrm{CD}_{14}{ }^{+} \mathrm{CD} 204^{+}$monocytes in peripheral blood were recognized as the M2-like tumor-associated 
monocytes, which were determined by flow cytometry (Fig. 2). As expected, the percentages of circulating $\mathrm{CD} 14^{+} \mathrm{CD} 204^{+}$monocytes from breast cancer patients $(11.02 \pm 2.74)$ were significantly higher than that from control groups $(7.50 \pm 1.73, p<0.001)$ (Fig. 3B). As conventional biomarkers, the plasma levels of CEA and CA15-3 in BC patients were significantly higher than that in control groups $(p=$ 0.027 and $p=0.003$, respectively) (Fig. 3 C and D). Therefore, our data showed that the frequency of circulating $\mathrm{CD}_{14}{ }^{+} \mathrm{CD} 204^{+}$M2-like monocytes and plasma HA were all significantly increased in breast cancer patients. Furthermore, there was a significant correlation between circulating M2-like monocytes and HA in breast cancer group (Spearman $r=0.404, p$ $<0.001$ ) (Table 2 and Fig. 3E).

Table 2. Spearman rank correlation among four biomarkers for distinguishing control group and breast cancer group

\begin{tabular}{|c|c|c|c|c|c|c|c|c|}
\hline & \multicolumn{4}{|c|}{ Control group } & \multicolumn{4}{|c|}{ Breast cancer group } \\
\hline & HA & M2\# & CEA & CA15-3 & HA & M2\# & CEA & CA15-3 \\
\hline HA & 1.000 & & & & 1.000 & & & \\
\hline M2\# & -0.050 & 1.000 & & & $0.404^{*}$ & 1.000 & & \\
\hline CEA & -0.023 & -0.005 & 1.000 & & 0.164 & 0.158 & 1.000 & \\
\hline CA15-3 & -0.084 & -0.160 & 0.133 & 1.000 & -0.011 & 0.044 & 0.218 & 1.000 \\
\hline
\end{tabular}

*: $p<0.05$; \#: circulating CD14+CD204+M2-like monocytes

\section{Circulating M2-like monocytes and plasma HA displays higher diagnostic values for breast cancer}

Receiver operating characteristic (ROC) analysis was applied to appraise the diagnostic values of the four biomarkers individually and their combination. When distinguishing breast cancers from controls, our results showed that the AUC (area under the ROC curve) values of circulating $\mathrm{CD} 14^{+} \mathrm{CD} 204^{+}$monocytes and plasma HA were 0.861 and 0.798 , respectively, higher than that of CEA (0.598) and CA15-3 (0.631) (Table 3 and Fig. 4A). Furthermore, logistic regression models were carried out to further evaluate the relationship between the four markers and breast cancer. As shown in Table 4, univariate logistic regression analysis presented that higher levels of circulating $\mathrm{CD}_{14}{ }^{+} \mathrm{CD} 204^{+}$M2-like monocytes $(\mathrm{OR}=$ 2.116; 95\% CI: $1.688-2.653 ; p<0.01)$ and plasma HA $(\mathrm{OR}=1.108 ; 95 \%$ CI: 1.065-1.152; $p<0.01)$ significantly predicted breast cancer. Correspondingly, CA15-3 $(\mathrm{OR}=1.107 ; 95 \%$ CI: 1.039-1.179; $p<0.05)$ and CEA (OR $=1.373 ; 95 \%$ CI: $1.017-1.853 ; p<0.05)$ also univariately predicted breast cancer versus controls. However, in multivariate logistic regression analysis, circulating M2-like monocytes, HA and CA15-3 but not CEA could significantly predict breast cancer.

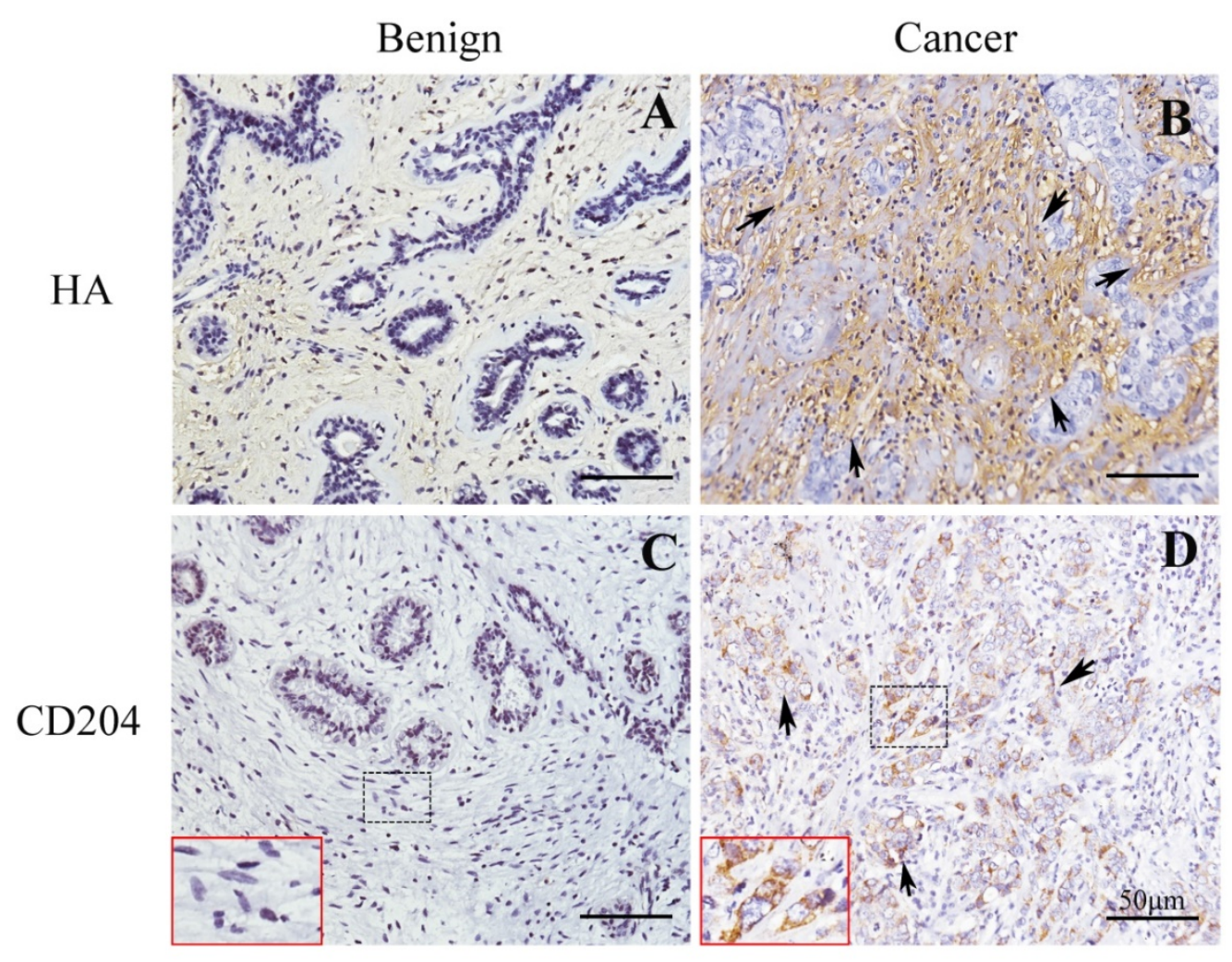

Figure 1. The expressions of M2-like macrophages and HA in breast cancer and benign tissues. Immunohistochemical assays confirmed the content of CD204+ M2-like macrophages and HA in breast cancer and benign breast disease. (A) and (B) HA expression in benign and cancer tissues, respectively. (C) and (D) CD204 expression in benign and cancer tissues, respectively. Shown were representative images of tissues from patients with breast cancer $(n=5)$ and benign disease $(n=$ 5). 
Table 3. Diagnostic values of HA, circulating CD14+CD204+ M2-like monocytes, CEA, CA15-3, and their combination for breast cancer

\begin{tabular}{|c|c|c|c|c|c|c|}
\hline \multirow[t]{2}{*}{ Markers } & \multirow[t]{2}{*}{ AUC } & \multirow[t]{2}{*}{$95 \% \mathrm{CI}$} & \multicolumn{4}{|l|}{ Sensitivity } \\
\hline & & & $90 \%$ specificity & $95 \% \mathrm{CI}$ & $80 \%$ specificity & $95 \% \mathrm{CI}$ \\
\hline HA & 0.798 & $0.733-0.863$ & $54.3 \%$ & $0.429-0.654$ & $60.5 \%$ & $0.490-0.863$ \\
\hline $\mathrm{CD}_{14}{ }^{+} \mathrm{CD} 204^{+}$ & 0.861 & $0.806-0.917$ & $71.6 \%$ & $0.605-0.811$ & $76.5 \%$ & $0.658-0.853$ \\
\hline CEA & 0.598 & $0.513-0.682$ & $14.8 \%$ & $0.079-0.245$ & $33.3 \%$ & $0.232-0.447$ \\
\hline CA15-3 & 0.631 & $0.548-0.714$ & $27.2 \%$ & $0.179-0.382$ & $39.5 \%$ & $0.288-0.510$ \\
\hline $\mathrm{CD} 14^{+} \mathrm{CD}_{204}{ }^{+}+\mathrm{HA}$ & 0.899 & $0.853-0.946$ & $74.1 \%$ & $0.631-0.832$ & $84.0 \%$ & $0.741-0.912$ \\
\hline CEA + CA15-3 & 0.639 & $0.557-0.722$ & $24.7 \%$ & $0.158-0.355$ & $32.1 \%$ & $0.222-0.434$ \\
\hline Combination of all & 0.908 & $0.863-0.954$ & $81.5 \%$ & $0.713-0.893$ & $86.4 \%$ & $0.770-0.930$ \\
\hline
\end{tabular}

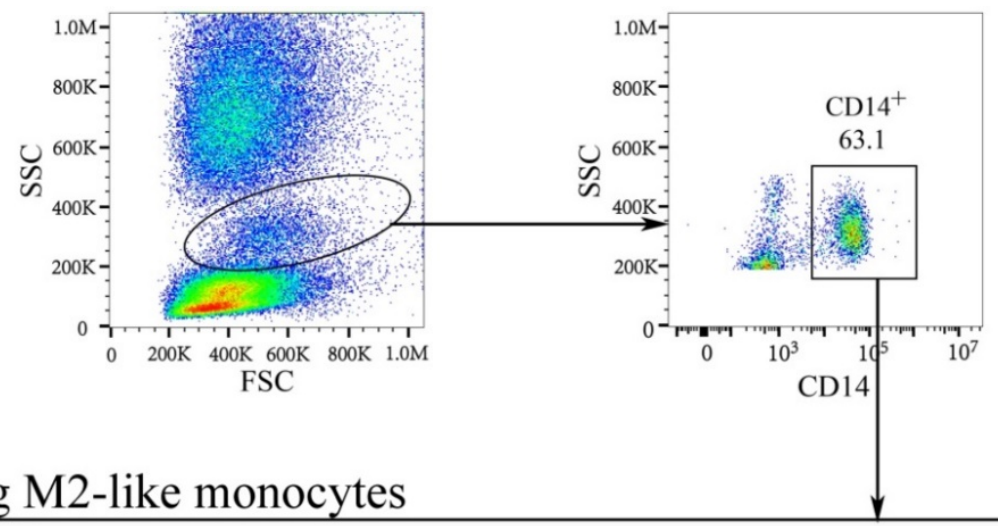

Circulating M2-like monocytes

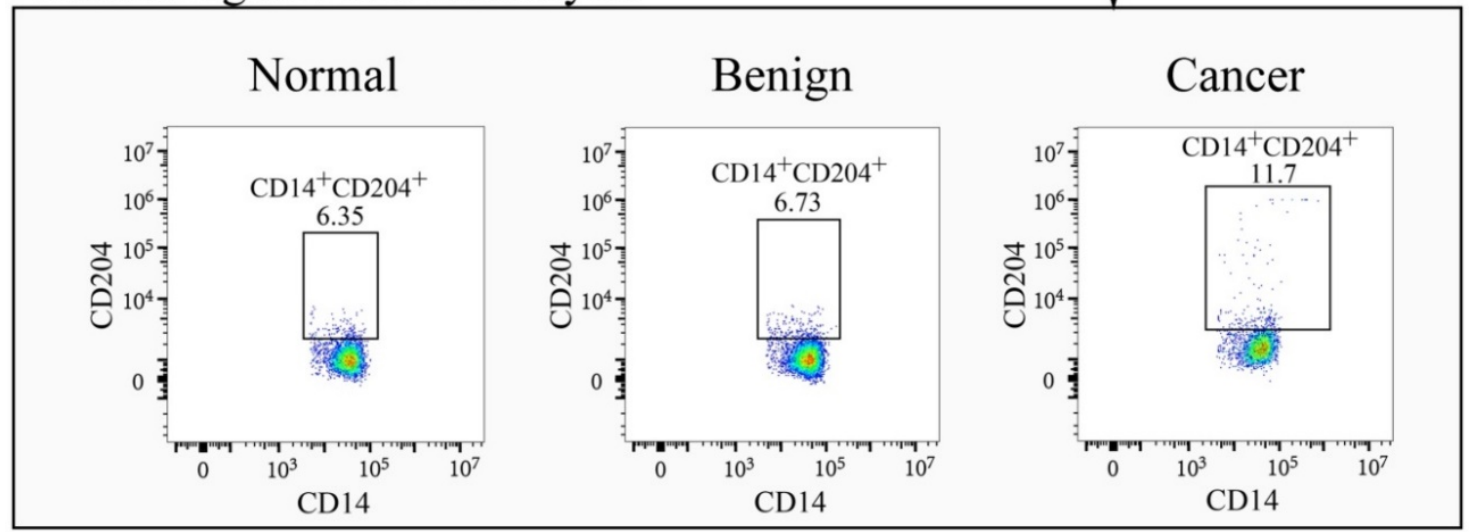

Figure 2. Characterization of circulating $\mathrm{CD14}+\mathrm{CD} 204+\mathrm{M} 2$-like monocytes in healthy controls, benign patients, and breast cancer patients. FSCmid/SSCmid fractions include mainly monocytes, which were gated initially on CD14+ cells. Subsequently, CD204+ M2-like monocytes were gated on CD14+ monocytes and the percentages of $\mathrm{CD} 14^{+} \mathrm{CD} 204^{+}$monocytes were determined.

In contrast to CEA and CA15-3, circulating $\mathrm{CD} 14^{+} \mathrm{CD} 204^{+}$monocytes and HA possessed higher sensitivity (Table 3 ). At specificity of $80 \%$, circulating $\mathrm{CD} 14^{+} \mathrm{CD} 204^{+}$monocytes and HA produced a sensitivity of $76.5 \%$ and $60.5 \%$, respectively, compared with CEA (33.3\%) and CA15-3 (39.5\%). Similarly, at $90 \%$ specificity, the sensitivities of circulating $\mathrm{CD} 14^{+} \mathrm{CD} 204^{+}$monocytes $(71.6 \%)$ and HA (54.3\%) were higher than CEA (14.8\%) and CA15-3 $(27.2 \%)$. In this study, on the basis of the logistic regression model, combining CA15-3 and CEA for differentiating breast cancer from controls produced a ROC curve with AUC of 0.639 (95\% CI: 0.557-0.722, $p$ $<0.05$ ), and the sensitivities were $32.1 \%$ at $80 \%$ specificity and $24.7 \%$ at $90 \%$ specificity. However, the AUC of the combination of circulating $\mathrm{CD} 144^{+} \mathrm{CD} 204^{+}$ monocytes and HA was 0.899 (95\% CI: 0.853-0.946, $p<$ 0.05 ), and the sensitivities reached $84.0 \%$ at $80 \%$ specificity and $74.1 \%$ at $90 \%$ specificity. Furthermore, as shown in Table 3 and Fig.4B, the AUC and sensitivity of the combination of circulating $\mathrm{CD} 14^{+} \mathrm{CD} 204^{+}$monocytes and HA were similar to those of all biomarkers combination (AUC $=0.908$, 95\% CI: $0.863-0.954 ; 86.4 \%$ at $80 \%$ specificity and $81.5 \%$ at $90 \%$ specificity). Collectively, our results indicated that circulating $\mathrm{CD} 14^{+} \mathrm{CD} 204^{+}$monocytes and HA might constitute a group of potential diagnostic marker for detection of breast cancer. 
A

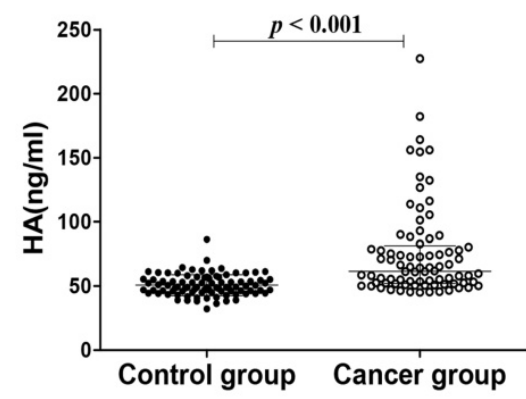

C

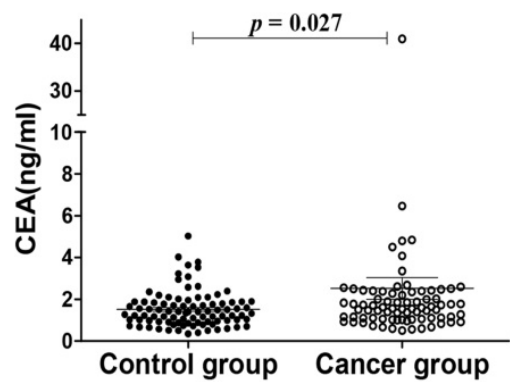

B

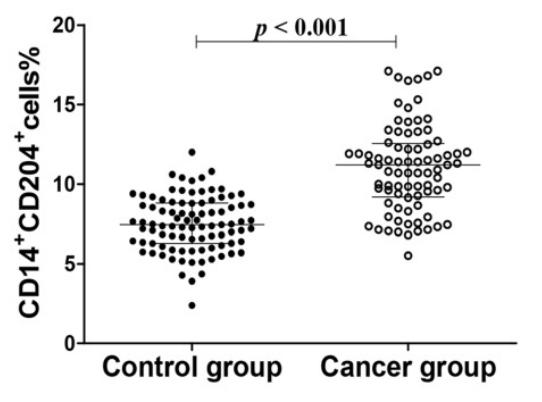

D

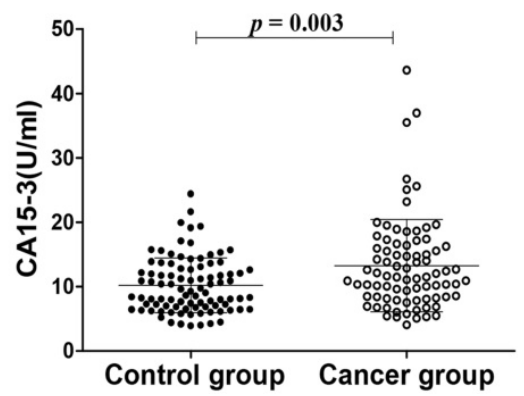

E

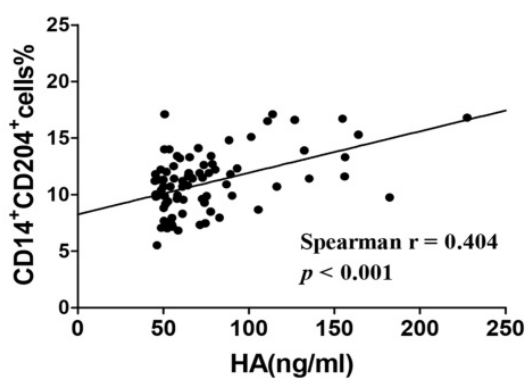

Figure 3. The levels of $\mathrm{HA}$, circulating $\mathrm{CD} 14+\mathrm{CD} 204+\mathrm{M} 2$-like monocytes, $\mathrm{CEA}$, and CA15-3 in patients with breast cancer and control subjects. (A) The levels of HA. (B) The percentages of circulating CD14+CD204+ M2-like monocytes. (C) and (D) The concentration of CEA and CA15-3, respectively. (E) The correlation between the percentages of circulating $\mathrm{CD} 14^{+} \mathrm{CD} 204^{+}$M2-like monocytes and the levels of plasma HA in BC patients.

A

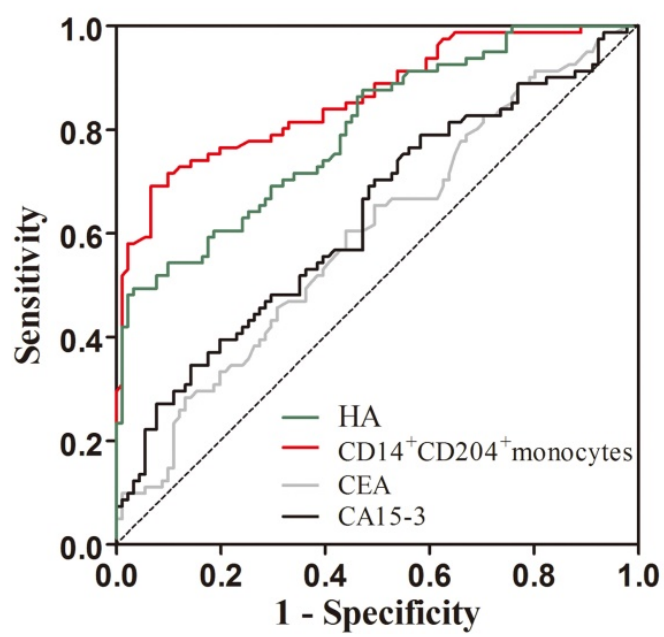

B

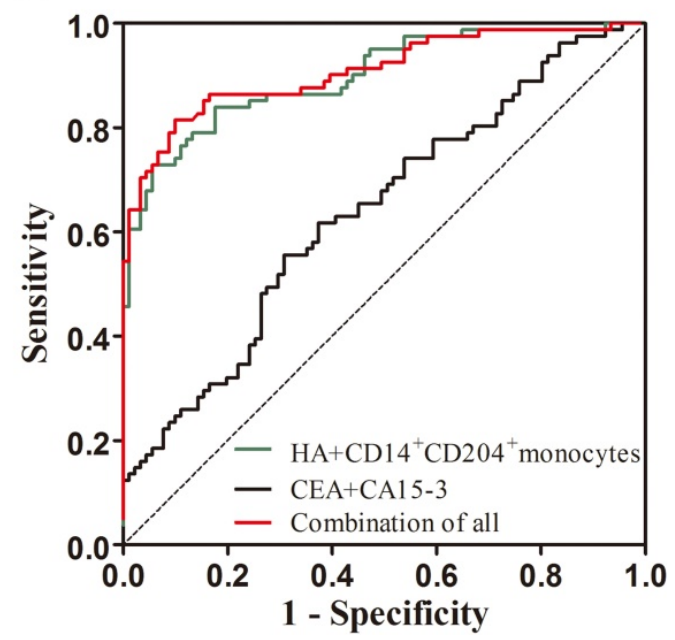

Figure 4. ROC analysis for the levels of individual biomarkers and their combination of them for comparing breast cancer to controls. (A) HA, circulating CD14+CD204+ M2-like monocytes, CEA, and CA15-3; (B) The combination of circulating CD14+CD204+ M2-like monocytes and HA, the combination of CEA and CA15-3, and the combination of all biomarkers.

\section{Correlation between the levels of all biomar- kers and clinical parameters of breast cancer}

The relationship of circulating $\mathrm{CD} 14^{+} \mathrm{CD} 204^{+}$ monocytes, HA, CA15-3, and CEA with patient characteristics were analyzed in this study. Our results showed that higher frequency of circulating CD14 ${ }^{+} \mathrm{CD} 204^{+}$M2-like monocytes were significantly associated with TNM stage, histological differentiation, and lymph node metastasis $(p<0.05)$.
Moreover, the frequency of $\mathrm{CD} 14^{+} \mathrm{CD} 204^{+}$M2-like monocytes in ER negative groups was higher than that in ER positive group $(p<0.05)$. However, besides the positive correlation between HA and histological differentiation, there was no association between HA and other clinical parameters, such as histology type, ER, and so on (Table 5). As shown in Table 5, our study found no connection between CEA, CA15-3 and clinical parameters $(p>0.05)$. 
Table 4. Results from logistic regression models

\begin{tabular}{llcccc}
\hline \multirow{2}{*}{ Markers } & \multicolumn{2}{c}{ Univariate } & & \multicolumn{2}{c}{ Multivariate } \\
\cline { 2 - 3 } \cline { 5 - 6 } & OR & $95 \% \mathrm{CI}$ & & OR & $95 \% \mathrm{CI}$ \\
\hline HA & $1.108^{*}$ & $1.065-1.152$ & & $1.092^{*}$ & $1.042-1.144$ \\
CD14+CD204+ & $2.116^{*}$ & $1.688-2.653$ & & $2.002^{*}$ & $1.538-2.606$ \\
CEA & $1.373^{*}$ & $1.017-1.853$ & & 1.278 & $0.833-1.963$ \\
CA15-3 & $1.107^{*}$ & $1.039-1.179$ & & $1.118^{*}$ & $1.011-1.236$ \\
\hline$*<0.05$ & & & &
\end{tabular}

\section{Correlation between the levels of all biomar- kers and clinical parameters of breast cancer}

The relationship of circulating $\mathrm{CD} 14^{+} \mathrm{CD} 204^{+}$ monocytes, HA, CA15-3, and CEA with patient characteristics were analyzed in this study. Our results showed that higher frequency of circulating $\mathrm{CD}_{14}{ }^{+} \mathrm{CD} 204^{+} \mathrm{M} 2$-like monocytes were significantly associated with TNM stage, histological differentiation, and lymph node metastasis $(p<0.05)$. Moreover, the frequency of $\mathrm{CD} 14^{+} \mathrm{CD} 204^{+}$M2-like monocytes in ER negative groups was higher than that in ER positive group $(p<0.05)$. However, besides the positive correlation between HA and histological differentiation, there was no association between HA and other clinical parameters, such as histology type, ER, and so on (Table 5). As shown in Table 5, our study found no connection between CEA, CA15-3 and clinical parameters $(p>0.05)$.

\section{Correlation between the levels of all biomar- kers and clinical parameters of breast cancer}

The relationship of circulating $\mathrm{CD} 14^{+} \mathrm{CD} 204^{+}$ monocytes, HA, CA15-3, and CEA with patient characteristics were analyzed in this study. Our results showed that higher frequency of circulating $\mathrm{CD}_{14}{ }^{+} \mathrm{CD} 204^{+} \mathrm{M} 2-$ like monocytes were significantly associated with TNM stage, histological differentiation, and lymph node metastasis $(p<0.05)$. Moreover, the frequency of $\mathrm{CD} 14^{+} \mathrm{CD} 204^{+}$M2-like monocytes in ER negative groups was higher than that in ER positive group $(p<0.05)$. However, besides the positive correlation between HA and histological differentiation, there was no association between HA and other clinical parameters, such as histology type, ER, and so on (Table 5). As shown in Table 5, our study found no connection between CEA, CA15-3 and clinical parameters $(p>0.05)$.

In the present study, we analyzed the percentage of circulating $\mathrm{CD}_{14}{ }^{+} \mathrm{CD} 204^{+}$M2-like monocytes in blood samples, and the frequency of which was significantly elevated in BC patients than that in control group. Similarly, plasma HA concentration detected in $\mathrm{BC}$ patients was also higher than control group. Notably, we found that the level of circulating $\mathrm{CD} 14^{+} \mathrm{CD} 204^{+}$M2-like monocytes was appeared to be positively correlated with plasma HA concentration in breast cancer. In contrast, there was no significant correlation between circulating M2-like monocytes and HA in control group. As indicated before, HA was an essential factor that induced the formation of M2-like phenotype besides other factors (e.g., IL-4, IL-13, M-CSF, and so on) in the tumor microenvironment $[7,12,13]$. Thus, our results suggested that tumor-derived HA and M2-like monocytes in peripheral blood could be used as potential biomarkers in diagnosis of breast cancer.

It is generally accepted that no single tumor biomarker will supply all necessary information for optimal diagnosis of cancer. The current tendency is to identify a multiple of biomarkers that can be used in combination to increase the diagnostic values. Up to now, very few tumor markers were clinically used for early discovery and diagnosis of breast cancer. The currently used tumor biomarkers CEA and CA15-3 were shown with low sensitivity in breast cancer that hindered its clinical application [3, 23, 24]. In agreement with previous studies, CA15-3 and CEA had relatively low sensitivity in breast cancer screening in our study. Nevertheless, our results demonstrated that circulating $\mathrm{CD} 14^{+} \mathrm{CD} 204^{+} \mathrm{M} 2$-like monocytes and HA displayed higher AUC and sensitivity at specificity of $80.0 \%$ and $90.0 \%$. Moreover, when combining circulating M2-like monocytes and HA with frequently-used markers to explore the diagnostic value of combination, we found that circulating M2-like monocytes and HA increased the diagnostic value with superior sensitivity and specificity for breast cancer diagnosis. Therefore, combination of circulating $\mathrm{CD} 14^{+} \mathrm{CD} 204^{+}$ M2-like monocytes and HA showed higher potential values than that of CEA and CA15-3.

We also found that higher levels of circulating $\mathrm{CD} 14^{+} \mathrm{CD} 204^{+}$M2-like monocytes were significantly correlated with lymph node metastasis and histological differentiation. Other studies indicated that circulating M2-like monocytes play an important role in the metastasis and growth of solid tumors [18, 25]. However, in this study, we found no significant association between plasma HA levels and lymph node metastasis and histological differentiation. The explanation may be that the measurement of plasma HA concentrations in our study is total HA. Previous study suggested that ECM degradation gives rise to the elevation of HA fragments which facilitate the tumor cell dissemination and metastasis [26]. In addition, our results revealed that the frequency of $\mathrm{CD}_{14}{ }^{+} \mathrm{CD} 204^{+} \mathrm{M} 2-$ like monocytes was increased in ER negative patients. Former studies showed that ER regulated the growth and differentiation of tumor cells and was positively correlated with the prognosis of breast cancer patients [27]. However, there was no relationship between CA15-3, CEA, and clinical 
parameters. Therefore, in contrast to CEA and CA15-3, circulating CD14 ${ }^{+} \mathrm{CD} 204^{+}$M2-like monocytes have the advantageous value of surveilling the progression of breast cancer.

In conclusion, this study indicated that there was a positive correlation between circulating $\mathrm{CD}^{+}{ }^{+} \mathrm{CD} 204^{+}$M2-like monocytes and HA in breast cancer, and the combination of them may represent a potential biomarker to screen breast cancer. However, our studies were carried out only in $\mathrm{CD} 14^{+}$monocytes and the assay of plasma HA was limited to total HA with small sample size. Therefore, further detailed analysis with large sample size is needed to verify our study.

Table 5. Correlation between the levels of HA, circulating CD14+CD204+ M2-like monocytes, CEA, and CA15-3 and clinicopathological characteristics of breast cancer patients

\begin{tabular}{|c|c|c|c|c|c|c|c|c|c|}
\hline Characteristics & NO. & $\begin{array}{l}\text { HA } \\
\text { Median }\left(\mathrm{P}_{25}-\mathrm{P}_{75}\right)\end{array}$ & $p$ & $\begin{array}{l}\mathrm{CD} 14^{+} \mathrm{CD} 204^{+}(\%) \\
(\mathrm{Mean} \pm \mathrm{SD})\end{array}$ & $p$ & $\begin{array}{l}\text { CEA } \\
\text { Median }\left(\mathrm{P}_{25}-\mathrm{P}_{75}\right)\end{array}$ & $p$ & $\begin{array}{l}\text { CA15-3 } \\
\text { Median }\left(\mathrm{P}_{25}-\mathrm{P}_{75}\right)\end{array}$ & $p$ \\
\hline \multicolumn{10}{|l|}{ Age(years) } \\
\hline$\leq 50$ & 38 & $55.22(51.10-78.50)$ & \multirow[t]{2}{*}{0.148} & $10.53 \pm 3.02$ & \multirow[t]{2}{*}{0.130} & $1.52(0.98-2.21)$ & \multirow[t]{2}{*}{0.237} & $13.60(10.16-17.69)$ & \multirow[t]{2}{*}{0.071} \\
\hline$>50$ & 43 & $66.98(58.06-86.88)$ & & $11.46 \pm 2.42$ & & $1.72(1.16-2.41)$ & & $10.21(8.10-15.00)$ & \\
\hline \multicolumn{10}{|l|}{ Tumor size (cm) } \\
\hline$\leq 2.0$ & 34 & $65.05(53.41-80.72)$ & \multirow[t]{2}{*}{0.528} & $10.75 \pm 2.42$ & \multirow[t]{2}{*}{0.448} & $1.45(1.01-1.88)$ & \multirow[t]{2}{*}{0.089} & $11.20(8.37-15.24)$ & \multirow[t]{2}{*}{0.639} \\
\hline$>2.0$ & 47 & $61.22(50.74-82.78)$ & & $11.22 \pm 2.96$ & & $1.61(1.15-2.50)$ & & $12.15(8.14-17.14)$ & \\
\hline \multicolumn{10}{|l|}{ TNM stage } \\
\hline $0-\mathrm{I}$ & 29 & $58.61(52.31-73.38)$ & \multirow{3}{*}{0.472} & $9.70 \pm 2.17$ & \multirow{3}{*}{$0.000^{*}$} & $1.35(0.94-2.10)$ & \multirow{3}{*}{0.113} & $10.30(8.33-14.47)$ & \multirow{3}{*}{0.267} \\
\hline II & 41 & $61.63(51.22-87.61)$ & & $11.35 \pm 2.69$ & & $1.76(1.38-2.41)$ & & $12.87(9.35-17.94)$ & \\
\hline III or higher & 11 & $70.36(50.60-154.7)$ & & $13.26 \pm 2.64$ & & $1.49(0.99-4.83)$ & & $12.63(6.72-17.40)$ & \\
\hline \multicolumn{10}{|l|}{ Histology type } \\
\hline Ductal & 54 & $61.43(53.34-80.86)$ & \multirow[t]{2}{*}{0.411} & $10.85 \pm 2.64$ & \multirow[t]{2}{*}{0.432} & $1.60(1.11-2.21)$ & \multirow[t]{2}{*}{0.912} & $12.04(8.21-17.34)$ & \multirow[t]{2}{*}{0.465} \\
\hline Others & 27 & $64.19(50.08-93.13)$ & & $11.36 \pm 2.96$ & & $1.49(1.03-2.47)$ & & $10.38(8.43-14.88)$ & \\
\hline \multicolumn{10}{|c|}{ Histology differentiation } \\
\hline Well-Moderate & 38 & $55.22(50.66-71.21)$ & \multirow[t]{2}{*}{$0.007^{*}$} & $9.39 \pm 2.14$ & \multirow[t]{2}{*}{$0.000^{*}$} & $1.54(1.01-2.50)$ & \multirow[t]{2}{*}{0.913} & $11.56(8.53-15.75)$ & \multirow[t]{2}{*}{0.962} \\
\hline Poor & 43 & $72.89(56.00-105.4)$ & & $12.46 \pm 2.40$ & & $1.59(1.10-2.02)$ & & $11.50(8.10-17.14)$ & \\
\hline \multicolumn{10}{|l|}{$\mathrm{N}$ status } \\
\hline N0 & 43 & $61.22(52.31-78.30)$ & \multirow[t]{2}{*}{0.623} & $9.82 \pm 2.43$ & \multirow[t]{2}{*}{$0.000^{*}$} & $1.54(1.08-2.39)$ & 0.233 & $10.92(8.07-15.23)$ & 0.075 \\
\hline N1-3 & 38 & $63.21(51.07-87.98)$ & & $12.38 \pm 2.45$ & & $1.66(1.13-2.36)$ & & $13.36(8.84-18.53)$ & \\
\hline ER & & & & & & & & & \\
\hline Positive & 53 & $59.65(52.31-74.89)$ & 0.207 & $9.94 \pm 2.32$ & $0.000^{*}$ & $1.70(1.10-2.42)$ & 0.410 & $11.50(8.46-16.34)$ & 0.792 \\
\hline Negative & 28 & $71.98(51.07-110.7)$ & & $13.07 \pm 2.28$ & & $1.50(1.04-1.99)$ & & $11.41(7.16-16.94)$ & \\
\hline PR & & & & & & & & & \\
\hline Positive & 48 & $61.17(51.46-77.84)$ & 0.651 & $10.57 \pm 2.71$ & 0.074 & $1.63(1.07-2.37)$ & 0.977 & $11.49(8.50-15.87)$ & 0.916 \\
\hline Negative & 33 & $64.19(52.20-95.73)$ & & $11.68 \pm 2.70$ & & $1.54(1.08-2.49)$ & & $12.05(7.19-17.61)$ & \\
\hline HER2 & & & & & & & & & \\
\hline Positive & 22 & $56.11(50.09-73.82)$ & 0.110 & $11.33 \pm 3.12$ & 0.533 & $1.31(1.01-2.44)$ & 0.585 & $10.65(7.76-16.63)$ & 0.381 \\
\hline Negative & 59 & $64.19(53.76-86.88)$ & & $10.91 \pm 2.61$ & & $1.61(1.11-2.38)$ & & $11.64(8.48-16.27)$ & \\
\hline Ki67 & & & & & & & & & \\
\hline$<20 \%$ & 36 & $65.39(52.95-77.30)$ & 0.540 & $10.48 \pm 2.44$ & 0.111 & $1.54(1.07-2.42)$ & 0.974 & $12.05(9.64-16.29)$ & 0.272 \\
\hline$\geq 20 \%$ & 45 & $59.65(50.78-84.83)$ & & $11.46 \pm 2.92$ & & $1.72(1.07-2.24)$ & & $11.50(4.07-16.47)$ & \\
\hline
\end{tabular}

${ }^{*}: p<0.05$. ER: Estrogen receptor, PR: Progesterone receptor, HER2: Human epidermal factor 2.

\section{Acknowledgments}

This work was supported by the National Natural Science Foundation of China (81272479, $81402419,81572821,81502490,81502491,81672843)$, the Natural Science Foundation of Shanghai Municipality (14YF1412200), the Program of Shanghai Leading Talents (2013-038), and the Shanghai Shen-Kang Hospital Development Center (SHDC22014004).

\section{Authors' contributions}

Boke Zhang, Manlin Cao, Guoliang Zhang, and Feng Gao contributed to the conception, design, and final approval of the submitted version. Yiqing He, Yiwen Liu, Jing $\mathrm{Xu}$, and Jiajie $\mathrm{Hu}$ performed the research and collected data. Boke Zhang, Cuixia Yang, and Yan Du analyzed the data. Boke Zhang, Guoliang Zhang, Feng Gao wrote and revised the manuscript. All authors read and approved the final manuscript.

\section{Competing Interests}

The authors have declared that no competing interest exists.

\section{References}

1. DeSantis C, Ma J, Bryan L, et al. Breast cancer statistics, 2013. CA Cancer J Clin. 2014; 64: 52-62.

2. Chen W, Zheng R, Baade PD, et al. Cancer statistics in China, 2015. CA Cancer J Clin. 2016; 66: 115-32.

3. Duffy MJ. Serum tumor markers in breast cancer: are they of clinical value? Clin Chem. 2006; 52: 345-51.

4. Sousa S, Brion R, Lintunen M, et al. Human breast cancer cells educate macrophages toward the M2 activation status. Breast Cancer Res. 2015; 17: 101. 
5. Italiani $\mathrm{P}$ and Boraschi D. From Monocytes to M1/M2 Macrophages: Phenotypical vs. Functional Differentiation. Front Immunol. 2014; 5: 514.

6. Laoui D, Movahedi K, Van Overmeire E, et al. Tumor-associated macrophages in breast cancer: distinct subsets, distinct functions. Int J Dev Biol. 2011; 55: 861-67.

7. Mantovani A, Sozzani S, Locati M, et al. Macrophage polarization: tumor-associated macrophages as a paradigm for polarized M2 mononuclear phagocytes. Trends Immunol. 2002; 23: 549-55.

8. Chavez-Galan L, Olleros ML, Vesin D, et al. Much More than M1 and M2 Macrophages, There are also $\mathrm{CD} 169(+)$ and $\mathrm{TCR}(+)$ Macrophages. Front Immunol. 2015; 6: 263.

9. Schwertfeger KL, Cowman MK, Telmer PG, et al. Hyaluronan, Inflammation, and Breast Cancer Progression. Frontiers in immunology. 2015; 6: 236.

10. Wu M, Cao M, He Y, et al. A novel role of low molecular weight hyaluronan in breast cancer metastasis. FASEB journal : official publication of the Federation of American Societies for Experimental Biology. 2015; 29: 1290-98.

11. Kobayashi N, Miyoshi S, Mikami T, et al. Hyaluronan deficiency in tumor stroma impairs macrophage trafficking and tumor neovascularization. Cancer Res. 2010; 70: 7073-83.

12. Tiainen S, Tumelius R, Rilla K, et al. High numbers of macrophages, especially M2-like (CD163-positive), correlate with hyaluronan accumulation and poor outcome in breast cancer. Histopathology. 2015; 66: 873-83.

13. Kuang $\mathrm{DM}, \mathrm{Wu} \mathrm{Y}$, Chen $\mathrm{N}$, et al. Tumor-derived hyaluronan induces formation of immunosuppressive macrophages through transient early activation of monocytes. Blood. 2007; 110: 587-95.

14. Zhang G, Guo L, Yang C, et al. A novel role of breast cancer-derived hyaluronan on inducement of M2-like tumor-associated macrophages formation. Oncoimmunology. 2016; 5: e1172154

15. Wyckoff JB, Wang Y, Lin EY, et al. Direct visualization of macrophage-assisted tumor cell intravasation in mammary tumors. Cancer Res. 2007; 67: 2649-56.

16. Roussos ET, Balsamo M, Alford SK, et al. Mena invasive (MenaINV) promotes multicellular streaming motility and transendothelial migration in a mouse model of breast cancer. J Cell Sci. 2011; 124: 2120-31.

17. Adams DL, Martin SS, Alpaugh RK, et al. Circulating giant macrophages as a potential biomarker of solid tumors. Proc Natl Acad Sci U S A. 2014; 111: 3514-19.

18. Maeda R, Ishii G, Neri S, et al. Circulating CD14+CD204+ cells predict postoperative recurrence in non-small-cell lung cancer patients. J Thorac Oncol. 2014; 9: 179-88.

19. Li C, Luo X, Lin $\mathrm{Y}$, et al. A Higher Frequency of CD14+ CD169+ Monocytes/Macrophages in Patients with Colorectal Cancer. PLoS One. 2015; 10: e0141817.

20. Jones K, Vari F, Keane C, et al. Serum CD163 and TARC as disease response biomarkers in classical Hodgkin lymphoma. Clin Cancer Res. 2013; 19: 731-42.

21. Condeelis J and Pollard JW. Macrophages: obligate partners for tumor cell migration, invasion, and metastasis. Cell. 2006; 124: 263-66.

22. Peng C, Wallwiener M, Rudolph A, et al. Plasma hyaluronic acid level as a prognostic and monitoring marker of metastatic breast cancer. Int J Cancer. 2016; 138: 2499-509.

23. Duffy MJ, Evoy D and McDermott EW. CA 15-3: uses and limitation as a biomarker for breast cancer. Clin Chim Acta. 2010; 411: 1869-74.

24. Cheung KL, Graves CR and Robertson JF. Tumour marker measurements in the diagnosis and monitoring of breast cancer. Cancer Treat Rev. 2000; 26: 91-102.

25. Cho HI, Jung JI, Lim DY, et al. Bone marrow-derived, alternatively activated macrophages enhance solid tumor growth and lung metastasis of mammary carcinoma cells in a Balb/C mouse orthotopic model. Breast Cancer Res. 2012; 14: R81.

26. Schmaus A, Klusmeier S, Rothley M, et al. Accumulation of small hyaluronan oligosaccharides in tumour interstitial fluid correlates with lymphatic invasion and lymph node metastasis. British journal of cancer. 2014; 111: 559-67.

27. Harvey JM, Clark GM, Osborne CK, et al. Estrogen receptor status by immunohistochemistry is superior to the ligand-binding assay for predicting response to adjuvant endocrine therapy in breast cancer. J Clin Oncol. 1999; 17: 1474-81. 